\title{
Stacking disorder by design: Factors governing the polytypism of silver iodide upon precipitation and formation from the superionic phase
}

\author{
Rachael L. Smith, ${ }^{a}$ Martin Vickers ${ }^{a}$ and Christoph G. Salzmann ${ }^{* a}$ \\ ${ }^{a}$ Department of Chemistry, University College London, 20 Gordon Street, London WC1H 0AJ, \\ United Kingdom. \\ * email: c.salzmann@ucl.ac.uk
}

\begin{abstract}
Silver iodide (AgI) is used for a wide range of applications from photocatalysis and antimicrobial coatings to photography and ice nucleation. By fitting powder X-ray diffraction patterns with DIFFaX, we show that AgI displays a strong tendency to form stacking-disordered materials. Its polytypism is determined by the silver cation to iodide molar ratio during precipitation. Under iodide-rich conditions, fully hexagonal $\beta$-AgI is obtained whereas a maximal percentage of cubic stacking of $80 \%$ is obtained at a 1:2 molar ratio in the silver cation-rich regime. These findings are explained on the basis of a concentration-dependent competition between kinetically and thermodynamically-favored adsorption processes. Furthermore, the previously reported memory effects observed upon transforming hexagonal and cubic AgI to the high-temperature superionic phase and back are now followed quantitatively. We propose that the memory effects originate from excess ions at the surfaces of AgI crystals that stabilize the pyroelectricity of AgI associated with hexagonal stacking. The ability to 'design' the polytypism of AgI by tuning the precipitation conditions provides a first example where the stacking disorder of a material can be controlled in a continuous fashion. Future studies will clarify if this design principle can be applied to other materials as well.
\end{abstract}




\section{Introduction}

Stacking-disordered materials consist of structurally well-defined layers that are stacked on top of one another in a disordered fashion. Naturally, stacking disorder is frequently found for layered materials, such as graphite ${ }^{1,2}$ or molybdenum sulfide, ${ }^{3}$ which display strong chemical bonding within their layers and weak interactions between them. However, there are also wellknown examples of stacking disorder, such as in ice $\mathrm{I}^{4-6}$ or diamond, ${ }^{7,8}$ where the chemical bonding is very similar if not identical within and between formal layer units. Countless other samples of stacking disorder exist ranging from complex inorganic materials ${ }^{9,10}$ and openframework materials ${ }^{11}$ to small-molecule pharmaceuticals. ${ }^{12-16}$ At present, there is a growing realization that the presence of stacking disorder and the associated phenomenon of polytypism can have profound consequences for the physical and chemical properties of the material in question. For example, in case of ice I, the extent of stacking disorder has been shown to affect its vapour pressure, ${ }^{17}$ crystal shapes, ${ }^{18}$ spectroscopic ${ }^{19}$ and light scattering properties ${ }^{18}$ as well as potentially surface chemistry. ${ }^{20}$ The hardness of diamond is thought to be influenced by the stacking disorder with fully hexagonal diamond potentially being significantly harder than its commonplace cubic counterpart. ${ }^{21,22}$ The solubility and hence bioavailability of pharmaceuticals could be affected by the extent of stacking disorder. In terms of material design, stacking disorder therefore offers the fascinating prospect of fine-tune the physical and chemical properties of materials if the stacking disorder can be controlled in a continuous fashion between the extreme polytypic endmembers.

Silver iodide (AgI) is one of inorganic chemistry's iconic materials, which famously displays superionic conductivity in its high-temperature $\alpha$-phase $(\operatorname{Im}-3 m) .{ }^{23}$ At low temperatures, two main polymorphs are known: the hexagonal wurtzite-type $\beta$-AgI $\left(P 6_{3} m c\right)^{24,25}$ and the cubic zinc-blende-type $\gamma$-AgI $(F-43 m) .{ }^{26}$ The structures of these two polytypes are shown in Figure 1(a,b). Both contain bilayers consisting of $\mathrm{Ag}^{+}$cations and $\mathrm{I}^{-}$anions, which are stacked on top of each in different fashions. In $\beta$-AgI, mirror images of the bilayers are stacked with the positions of cations and anions swapped (Figure 1(a)). The structure of $\gamma$-AgI can be built-up by placing identical bilayers on top of one another and performing diagonal shifts within the plane of the bilayers (Figure 1(b)). Consequently, the unit cells of $\beta$-AgI and $\gamma$-AgI contain two or three bilayers, respectively, and their structures are described as $2 \mathrm{H}$ or $3 \mathrm{C}$ in the Ramsdell notation for describing polytypes. ${ }^{27-29}$ 


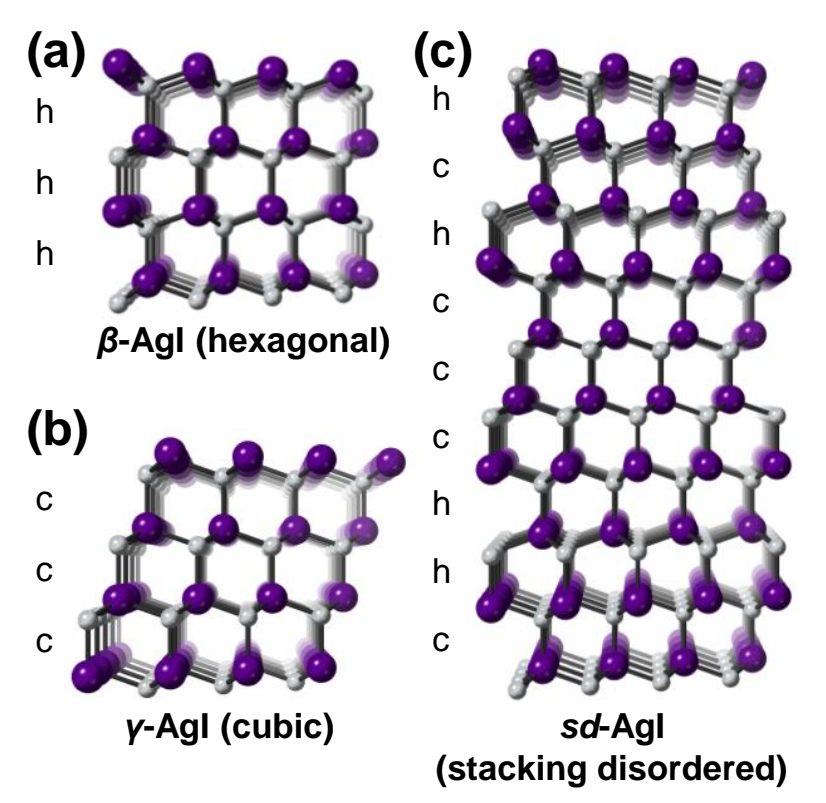

Figure 1 Structures of (a) hexagonal $\beta$-AgI, (b) cubic $\gamma$-AgI and (c) a possible stacking sequence of stacking disordered $\mathrm{AgI} . \mathrm{Ag}^{+}$cations and $\mathrm{I}^{-}$anions are represented by gray and purple spheres, respectively. Hexagonal and cubic stacking events are labelled with " $\mathrm{h}$ " and "c".

From the application point of view, AgI can be used in black and white photography ${ }^{30}$ it is an

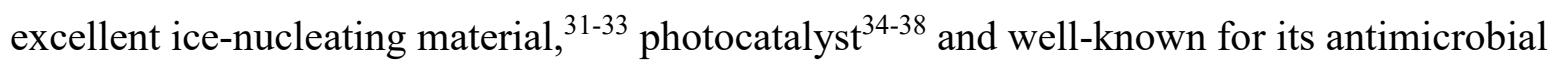
properties. ${ }^{39}$ Furthermore, tremendous efforts have been made to stabilize the superionic $\alpha$-phase at room temperature. ${ }^{40-44}$

Although $\gamma$-AgI was originally believed to be more stable than $\beta$-AgI at room temperature, ${ }^{45}$ it is now generally recognized that $\beta-\mathrm{AgI}$ is the most stable phase at ambient pressure below $147^{\circ} \mathrm{C}$ where the phase transition to $\alpha-\mathrm{AgI}$ takes place.$^{29} \mathrm{In}$ addition to $\beta$ and $\gamma-$ AgI, a range of complex polytypes have been found mainly on the basis of single-crystal X-ray diffraction including the $4 \mathrm{H}, 7 \mathrm{H}, 8 \mathrm{H}, 12 \mathrm{H}_{\mathrm{a}}, 12 \mathrm{H}_{\mathrm{b}}$ and $16 \mathrm{H}$ polytypes but also randomly stacking disordered AgI ( $s d-\mathrm{AgI}){ }^{27-29}$ A possible stacking sequence of $s d-\mathrm{AgI}$ is shown in Figure 1(c).

Several recipes for the preparation of the various AgI polytypes as well as the growth of single crystals of $\beta$-AgI have been reported. ${ }^{27-29} \mathrm{An}$ excess of iodide ions during the crystallization of $\mathrm{AgI}$ leads to $\beta$ - $\mathrm{AgI}^{24,45-47}$ whereas an excess of silver ions seems to favour the formation of $\gamma$-AgI. ${ }^{25,48,49}$ However, the determination of the cubicity of a sample $\Phi_{\mathrm{c}}$, i.e. the fraction of cubic stacking, is difficult. ${ }^{29}$ Single-crystal diffraction data can only be used to 
identify highly ordered polytypes ${ }^{27-29}$ and often it has been found that the crystal under investigation was not representative of the entire sample. ${ }^{25}$ The analysis of powder diffraction data is typically complicated by the presence of diffuse diffraction features, which are caused by the stacking disorder. A traditional quantitative Rietveld analysis using, for example, a physical mixture of $\beta$ - $\mathrm{AgI}$ and $\gamma$-AgI is therefore not possible. The ambiguity of polytype identification from NMR data has also been discussed. ${ }^{29}$

An interesting aspect of the polytypism of AgI is the 'memory effect' observed when $\beta / \gamma$ AgI is transformed to $\alpha$ - $\mathrm{AgI}$ and back again. ${ }^{45,50,51} \mathrm{In} \alpha$-AgI, the iodide anions are packed in a body-centered cubic fashion, and the silver cations have high mobilities and are distributed over various crystal sites. ${ }^{52,53}$ Accordingly, the information about the cubicity of the original $\beta / \gamma-\mathrm{AgI}$ sample should be lost once transformed to $\alpha$-AgI. Yet, experimentally, it has been observed that both $\beta$-AgI and $\gamma$-AgI have tendencies to reform following the transformation to $\alpha$-AgI. ${ }^{45,50,51} \mathrm{As}$ a possible explanation for this memory effect, it has been suggested that the $\mathrm{Ag}^{+}$ions are not randomly distributed in $\alpha$ - $\mathrm{AgI}$ thereby retaining proximity to the $\mathrm{Ag}^{+}$sites in the respective lowtemperature polymorphs. ${ }^{50}$ Later, it was suggested that pure and defect-free $\beta$-AgI transforms to $\alpha-\mathrm{AgI}$ at a higher temperature than $\gamma-\mathrm{AgI}$, and that this hysteresis may be responsible for the observed memory effect. ${ }^{51}$

Overall, it is clear that there is a need for more refined quantitative methods to characterize the polytypism of AgI. ${ }^{29}$ Here we use the DIFFaX approach ${ }^{54}$ in combination with our MCDIFFaX program $^{5,8}$ to model the X-ray diffraction patterns of a range of AgI samples. DIFFaX enables us to include the diffuse scattering that arises from stacking disorder in the fitting of the diffraction patterns and to hence obtain accurate values for the cubicities. On this basis, we aim to gain insights into the factors that govern the formation of either $\beta$ - $\mathrm{AgI}$ or $\gamma$-AgI upon precipitation but also to follow the memory effect coming from the $\alpha$-phase quantitatively.

\section{Experimental Section}

Preparation of silver iodide samples

A large magnetic stirrer bar was placed in an Erlenmeyer flask, vigorous stirring was started and $5 \mathrm{~mL}$ each of $\mathrm{NaI}$ and $\mathrm{AgNO}_{3}$ solutions were added simultaneously resulting in the immediate precipitation of light-yellow silver iodide (AgI) according to $\mathrm{NaI}(\mathrm{aq})+\mathrm{AgNO}_{3}(\mathrm{aq}) \rightarrow \mathrm{AgI}(\mathrm{s})+$ 
$\mathrm{NaNO}_{3}(\mathrm{aq})$. The NaI solutions were prepared by dissolving $\mathrm{NaI}$ in deionized water while the silver nitrate was dissolved in $0.1 \mathrm{M}$ nitric acid to prevent the possible formation of silver oxide at higher $\mathrm{pH}$ values. The concentrations of both $\mathrm{NaI}$ and $\mathrm{AgNO}_{3}$ in the two initial solutions as well as the corresponding molar ratios between the $\mathrm{Ag}^{+}$cations and $\mathrm{I}^{-}$anions after mixing, $x\left(\mathrm{Ag}^{+}\right)$, are given in Table 1. The AgI precipitates were recovered by filtration using $0.2 \mu \mathrm{m}$ track-edged polycarbonate membranes, washed with water and ethanol on the filter membrane, and then dried in a desiccator. Due to the light-sensitive nature of AgI, the samples were handled as far as practicable under a red LED light.

Table 1. Concentrations of $\mathrm{AgNO}_{3}$ and $\mathrm{NaI}$ in the initial solutions as well as the molar ratios between $\mathrm{Ag}^{+}$and $\mathrm{I}^{-}$ions.

\begin{tabular}{|c|c|c|}
\hline$c\left(\mathrm{AgNO}_{3}\right) / \mathrm{mol} \mathrm{L}^{-1}$ & $c(\mathrm{NaI}) / \mathrm{mol} \mathrm{L}^{-1}$ & $x\left(\mathrm{Ag}^{+}\right)=c\left(\mathrm{Ag}^{+}\right) / c\left(\mathrm{I}^{-}\right)$ \\
\hline 10 & 1 & 10 \\
\hline 5 & 1 & 5 \\
\hline 2 & 1 & 2 \\
\hline 1.5 & 1 & 1.5 \\
\hline 1 & 1 & 1 \\
\hline 1 & 1.5 & 0.667 \\
\hline 1 & 2 & 0.5 \\
\hline 1 & 5 & 0.2 \\
\hline 1 & 7 & 0.143 \\
\hline
\end{tabular}

A few selected samples were heated to $200^{\circ} \mathrm{C}$ at $10^{\circ} \mathrm{C} \mathrm{min}^{-1}$ in a Carbolite MTF 1200 horizontal tube furnace to achieve the transition to $\alpha$-AgI. The samples were held at $200^{\circ} \mathrm{C}$ for $1 \mathrm{~h}$ to allow thermal equilibration. Following this, the samples were either quickly extracted from the furnace and cooled outside the furnace, or cooled at $1{ }^{\circ} \mathrm{C} \mathrm{min}^{-1}$ in the furnace to room temperature. By attaching a thermocouple to the crucible, the cooling rate for the quick extraction was estimated to be greater than $10^{\circ} \mathrm{C} \mathrm{min}^{-1}$. 
Powder X-ray diffraction

Samples were characterised with powder X-ray diffraction using a Stoe StadiP transmission instrument ( $\mathrm{Mo} \mathrm{K} \alpha_{1}$ radiation, $50 \mathrm{kV}, 30 \mathrm{~mA}$ ) in the $2 \theta$ range from 2 to $40^{\circ}$ with $0.5^{\circ}$ detector steps every $5 \mathrm{~s}$ giving a step size of $0.015^{\circ}$. The powders were sandwiched between two thin cellulose acetate foils and rotated during the measurements. Additionally, samples were mounted in $0.5 \mathrm{~mm}$ borosilicate glass capillaries, rotated in the beam and heated using a Stoe furnace during data collection. Heating ramps were from room temperature to $200^{\circ} \mathrm{C}$ at $2^{\circ} \mathrm{C} \mathrm{min}^{-1}$ in $10^{\circ} \mathrm{C}$ steps with patterns being collected at each step followed by immediate cooling back to room temperature where a final pattern was collected. In a second set of experiments, heating ramps were carried out to $140^{\circ} \mathrm{C}$ at $2^{\circ} \mathrm{C} \mathrm{min}^{-1}$ in $10^{\circ}$ steps followed by annealing for either 2 or 10 hours and then cooling to room temperature.

\section{MCDIFFaX analysis}

The recorded diffraction patterns were baseline corrected using shifted Chebyshev background functions in the GSAS software. ${ }^{55}$ The diffraction data was then fitted with the MCDIFFaX software by refining $2^{\text {nd }}$ order stacking probabilities, lattice parameters, peak-profile parameters ( $u, v, w$ and Gaussian / Lorentzian ratio) and thermal displacement parameters of $\mathrm{Ag}^{+}{\text {and } \mathrm{I}^{-} .56}^{-6}$ During a typical refinement, a starting value of 0.5 was used as a starting value for the zero-order stacking probability, and the $1^{\text {st }}$ and $2^{\text {nd }}$ order stacking probabilities were successively introduced during the refinement once the fit had converged using the lower-order stacking probabilities. A detailed description of the MCDIFFaX program as well as the equations needed for the calculations of the $1^{\text {st }}$ order probabilities from the $2^{\text {nd }}$ order probabilities as well as the cubicity from the $1^{\text {st }}$ order probabilities are given in ref. 8 .

\section{Results and Discussion}

Silver iodide samples were prepared by mixing aqueous solutions of $\mathrm{AgNO}_{3}$ and $\mathrm{NaI}$ under vigorous stirring ensuring rapid mixing of the two solutions. Light-yellow precipitates of AgI formed instantaneously, which were then isolated after filtration, washing and drying. These precipitation experiments were carried out for a range of different $\mathrm{Ag}^{+}$and $\mathrm{I}^{-}$concentrations in the initials solutions resulting in different $\mathrm{Ag}^{+}$to $\mathrm{I}^{-}$molar ratios, $x\left(\mathrm{Ag}^{+}\right)$, during precipitation. Since equal volumes of the $\mathrm{AgNO}_{3}$ and $\mathrm{NaI}$ solutions were mixed, the $\mathrm{Ag}^{+}$to $\mathrm{I}^{-}$molar ratios during the 
precipitation can be calculated from the $\mathrm{Ag}^{+}$and $\mathrm{I}^{-}$concentrations of the initial solutions according to $x\left(\mathrm{Ag}^{+}\right)=c\left(\mathrm{Ag}^{+}\right) / c\left(\mathrm{I}^{-}\right)(c f$. Table 1).

The polytypism of the AgI samples was found to depend strongly on the $\mathrm{Ag}^{+}$to $\mathrm{I}^{-}$molar ratio. Figure 2(a) shows the experimental diffraction patterns of AgI samples prepared in the $x\left(\mathrm{Ag}^{+}\right)$range from 1:7 to 10:1. For comparison, the calculated diffraction patterns of hexagonal $\beta$ AgI and cubic $\gamma$-AgI are shown in Figure 2(b). The diffraction patterns of the 1:7 and 1:5 samples were consistent with fully hexagonal $\beta$-AgI. However, upon further decreasing the amount of $\mathrm{I}^{-}$ anions in the precipitation mixtures, the diffraction patterns show clear signs of stacking disorder in the form of diffuse scattering around, for example, the "trident" feature at $~ 11$ degrees.

Using our MCDIFFaX program, 5, 8 all experimental diffraction patterns were fitted using up to $2^{\text {nd }}$ order memory effects in the stacking sequences. The resulting MCDIFFaX fits and the obtained cubicities, $\Phi_{c}$, of the various samples are shown in Figure 2(a). As mentioned earlier, the cubicity reflects the percentage of cubic stacking present in a sample which is related to its hexagonality, $\Phi_{\mathrm{h}}$, by $1-\Phi_{\mathrm{c}}$.

Figure 3(a) shows that the cubicity depends on the $\mathrm{Ag}^{+}$to $\mathrm{I}^{-}$molar ratio in a systematic fashion. Purely hexagonal $\beta$-AgI forms only in very iodide-rich solutions. A sharp rise in cubicity is observed as the relative amount of $\mathrm{I}^{-}$is reduced and at a 1:1 molar ratio, the sample displayed a cubicity of $58 \%$. A maximum in the cubicity of $81 \%$ was found for a $1: 2$ molar ratio in the $\mathrm{Ag}^{+}$rich domain. After this, the cubicity decreases again. Yet, even at a 1:10 excess of $\mathrm{Ag}^{+}$, the sample was still found to be considerably stacking disordered. Therefore, unlike the precipitation under large $\mathrm{I}^{-}$excesses, the formation of fully hexagonal AgI was not observed in our experiments for a large excess of $\mathrm{Ag}^{+}$.

A possible explanation of the 'bell-shaped' trend in Figure 3(a) can be given by considering the thermodynamics and kinetics of different adsorption processes onto the precipitating AgI. Adsorption of ions onto sites that lead to the formation of hexagonal stacking must be favored thermodynamically since $\alpha$-AgI is the thermodynamically stable phase. In turn, the adsorption onto sites that lead to cubic stacking must therefore be thermodynamically less favorable. Yet, the fact that cubic stacking is observed at all, means that the adsorption onto "cubic sites" is kinetically favored and therefore faster compared to adsorption onto "hexagonal sites". 


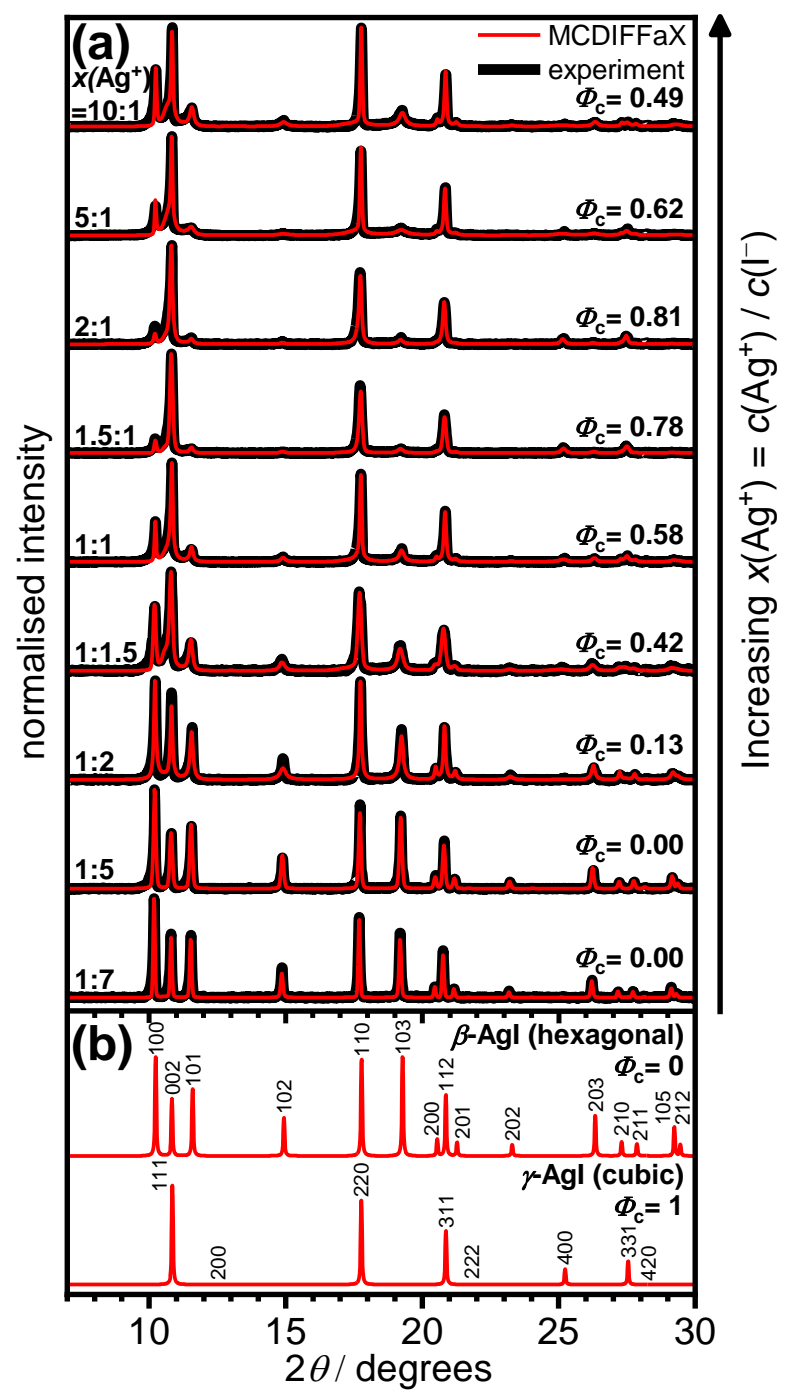

Figure 2 (a) X-ray diffraction patterns (Mo K $\alpha_{1}$ ) of $\mathrm{AgI}$ samples prepared using different $\mathrm{Ag}^{+}$to $\mathrm{I}^{-}$molar ratios, $x\left(\mathrm{Ag}^{+}\right)$, upon precipitation. The experimental data and MCDIFFaX fits are shown as thick black lines and thin red lines, respectively. (b) Simulated X-ray diffraction patterns of $\beta$ AgI and $\gamma$-AgI. The cubicities, $\Phi_{c}$, are given for all diffraction patterns. 

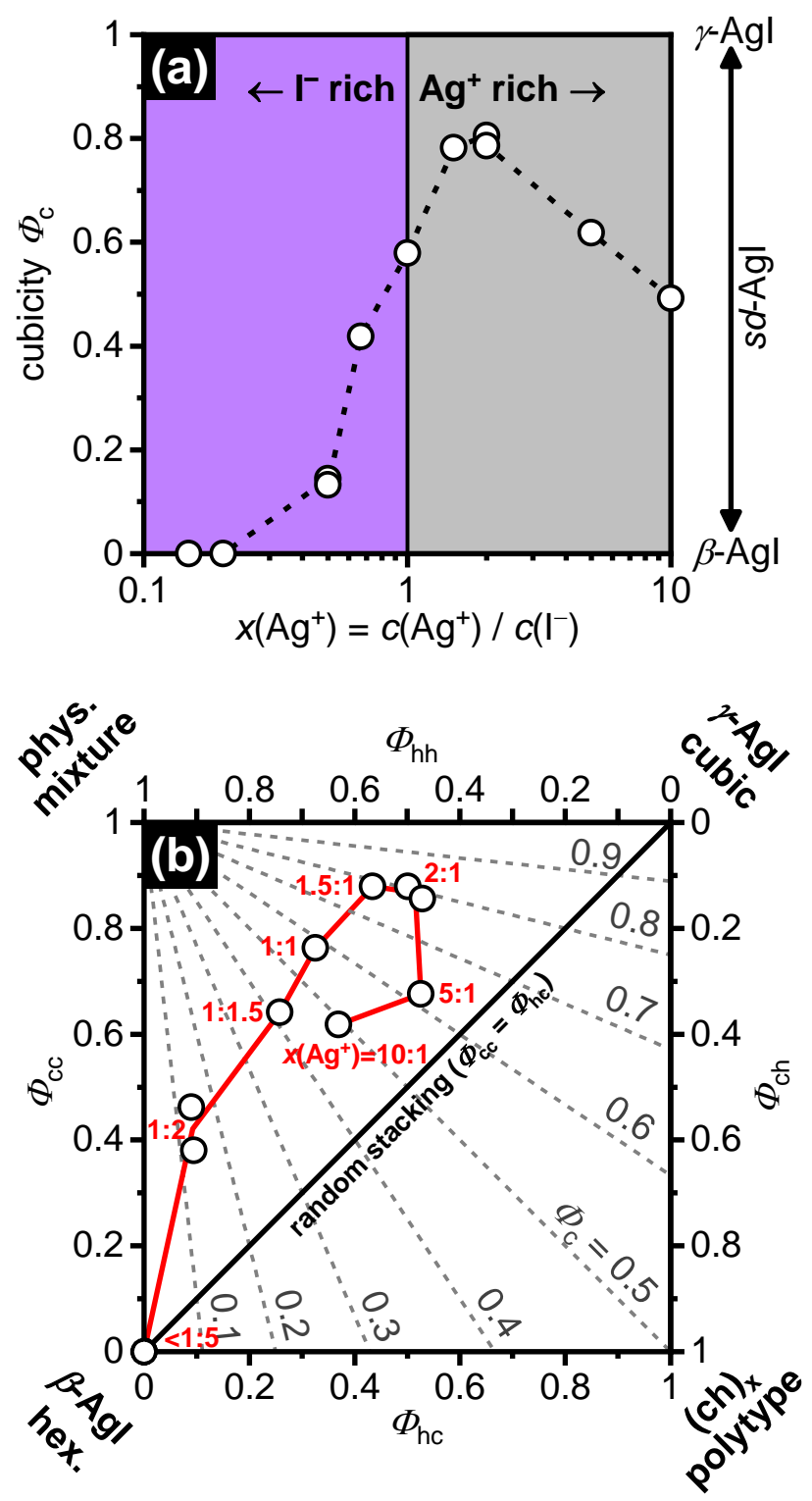

Figure 3 Characteristics of stacking disorder in $\mathrm{AgI}$ depending on the $\mathrm{Ag}^{+}$to $\mathrm{I}^{-}$molar ratio upon precipitation. (a) Changes in cubicity with the $\mathrm{Ag}^{+}$to $\mathrm{I}^{-}$molar ratio. (b) First-order memory effects in the stacking sequences indicated on a "stackogram". 5,8 Dashed gray lines indicate constant cubicities whereas the diagonal solid line reflects random stacking.

At roughly equal concentrations of the ions, the precipitation is therefore dominated by the formation of cubic stacking. The fast adsorption processes take place predominately and, once adsorption has taken place onto a "cubic" site, the ion is locked into place by the also fast adsorption of ions of opposite charge. 
If large excesses of one of the ions are present, then the excess ionic species compete for the various adsorption sites and cannot be as easily "fixated" by the minority species due to its comparatively low concentration. The result is that the energetically most favorable adsorption onto "hexagonal" sites takes place and it seems even possible that an "accidental" adsorption onto a "cubic" site can be reversed due to the energy gained by adsorption onto a neighboring "hexagonal" site. The minority ionic species may still adsorb onto "cubic" sites. However, as the majority species compete for adsorption, it seems possible that the minority species can diffuse on the surface to a more favorable "hexagonal" site.

In line with the high mobility of $\mathrm{Ag}^{+}$ions in $\alpha$ - $\mathrm{AgI}$, it seems sensible to assume that the smaller $\mathrm{Ag}^{+}$cations also display a higher mobility at the precipitation interface compared to the larger $\mathrm{I}^{-}$anions. This would explain why (i) it is possible to obtain fully hexagonal $\beta$-AgI in the $\mathrm{I}^{-}$ -rich / $\mathrm{Ag}^{+}$-deficient precipitation regime, (ii) the cubicity maximum is not located precisely at a 1:1 molar ratio of the ions and (iii) it is not possible to obtain fully hexagonal $\beta-\mathrm{AgI}$ in the $\mathrm{Ag}^{+}-$ rich regime due to the lower surface mobility of the iodide anions. In addition to the adsorption effects discussed here, it is noted that the formation of hexagonal $\beta$-AgI from iodide-rich solutions could also be due to the existence of Ag-I complexes in solution. ${ }^{45}$

The memory effects present in the stacking sequences of the various AgI samples are illustrated by the "stackogram" shown in Figure 3(b). Here, the first-order stacking probabilities, $\Phi_{\mathrm{cc}}$ and $\Phi_{\mathrm{hc}}$, obtained from the MCDIFFaX fits are shown. For example, $\Phi_{\mathrm{hc}}$, reflects the probability of a cubic stacking event following hexagonal stacking. The corresponding probability of hexagonal stacking after hexagonal stacking, $\Phi_{\mathrm{hh}}$, can be calculated from $\Phi_{\mathrm{hh}}=\Phi_{\mathrm{hc}}-1$. Accordingly, the four corners of the stackogram indicate fully cubic $\gamma-\operatorname{AgI}\left(\Phi_{\mathrm{cc}}=\Phi_{\mathrm{hc}}=1\right)$, fully hexagonal $\beta-\operatorname{AgI}\left(\Phi_{\mathrm{ch}}=\Phi_{\mathrm{hh}}=1\right)$, a physical mixture of $\beta-\operatorname{AgI}$ and $\gamma-\operatorname{AgI}\left(\Phi_{\mathrm{cc}}=\Phi_{\mathrm{hh}}=1\right)$ and finally the perfectly alternating $4 \mathrm{H}$ polytype $\left(\Phi_{\mathrm{ch}}=\Phi_{\mathrm{hc}}=1\right)$. Lines of constant cubicity emanate from the physical mixture corner as indicated by the gray dashed lines whereas the line of random stacking, where $\Phi_{\mathrm{cc}}=\Phi_{\mathrm{ch}}$ and stacking is therefore independent of the previous stacking history, is shown by the solid diagonal line.

All samples obtained in this study display first-order stacking probabilities above the random line which means that there is generally tendency in AgI to repeat the previous type of stacking. In addition to the competition between thermodynamic and kinetic processes at the precipitation 
interface, there seems to be some sort of self-templating effect based on previous stacking events. Such a behavior has also been previously observed for stacking-disordered ice and diamond. ${ }^{5,8,57}$ As can be seen from Figure 3(b), the trend for clustering of like stacking events is more pronounced for the samples prepared in the iodide-rich regime. This makes sense, since in this regime, highly extended regions of hexagonal stacking eventually form as the excess of $\mathrm{I}^{-}$anions is increased.

Based on our results, it is clear that the nomenclature to describe the structures of $\mathrm{AgI}$ samples must be extended. We proposed to reserve $\beta$-AgI and $\gamma$-AgI for the fully hexagonal and cubic polytypes, respectively, and use stacking-disordered silver iodide ( $s d-\mathrm{AgI})$ for all polytypic structures between the two endmembers. In the context of the high-pressure phases of AgI, $\beta$-AgI and $\gamma$-AgI are often referred to as AgI-II and II', respectively. ${ }^{58,59}$ We suggest that in this context, AgI-II $h$, AgI-II $c$ and AgI-IIsd should be used.

Using our MCDIFFaX approach for the characterization of stacking disorder in AgI, we now turn to investigating the 'memory effect' after transforming to the body-centered $\alpha$-AgI. ${ }^{45,50,51}$ The fact that the information on the original cubicity of a sample seems to somehow 'survive' the transformation to the $\alpha-\mathrm{AgI}$ has been previously attributed to tiny domains of the low-temperature phases remaining in the $\alpha-\mathrm{AgI},{ }^{45}$ not entirely randomly occupied $\mathrm{Ag}^{+}$positions in $\alpha-\mathrm{AgI}^{50}$ as well as two different phase transitions temperatures of $\beta / \gamma \mathrm{AgI} .{ }^{51}$

Figure 4(a) shows a typical sequence of diffraction patterns recorded upon heating $s d$-AgI with $\Phi_{\mathrm{c}}=80 \%$ from room temperature to $200^{\circ} \mathrm{C}$. Consistent with the literature, ${ }^{29}$ a sudden phase transition to $\alpha$-AgI is observed at around $147^{\circ} \mathrm{C}$. The $\alpha$ - $\mathrm{AgI}$ was then either cooled slowly at $1^{\circ} \mathrm{C}$ $\mathrm{min}^{-1}$ or quickly at a cooling rate greater than $10^{\circ} \mathrm{C} \mathrm{min}^{-1}$ back to room temperature. The cubicities of the recovered samples were 46 and $42 \%$, respectively. In a second set of experiments, fully hexagonal $\beta$-AgI samples were transformed to $\alpha$-AgI and subjected to the same cooling procedures. Here, cubicities of 27 and $34 \%$ were obtained after cooling at 1 and $>10^{\circ} \mathrm{C} \mathrm{min}^{-1}$, respectively. The fact that slow-cooling led to more hexagonal samples for both starting materials illustrates the general thermodynamic drive force towards $\beta$-AgI. Furthermore, based on our experiments, the previously observed memory effects can be confirmed. ${ }^{45,50,51}$ However, now that we have analyzed the memory effect quantitatively in terms of the cubicities of the sample, it is fair to state that the memory effect is probably somewhat less pronounced than previously thought. The original difference in cubicity of the two starting materials was $80 \%$ which decreased to either 
12 or $15 \%$ depending on whether cooling rates of $>10$ or $1{ }^{\circ} \mathrm{C} \min ^{-1}$ were used. Of course, the somewhat less pronounced memory effects observed here may be connected with the fact that our samples were heated to $200^{\circ} \mathrm{C}$, whereas $180^{\circ} \mathrm{C}$ was typically the highest temperature in previous studies. $^{45,50,51}$
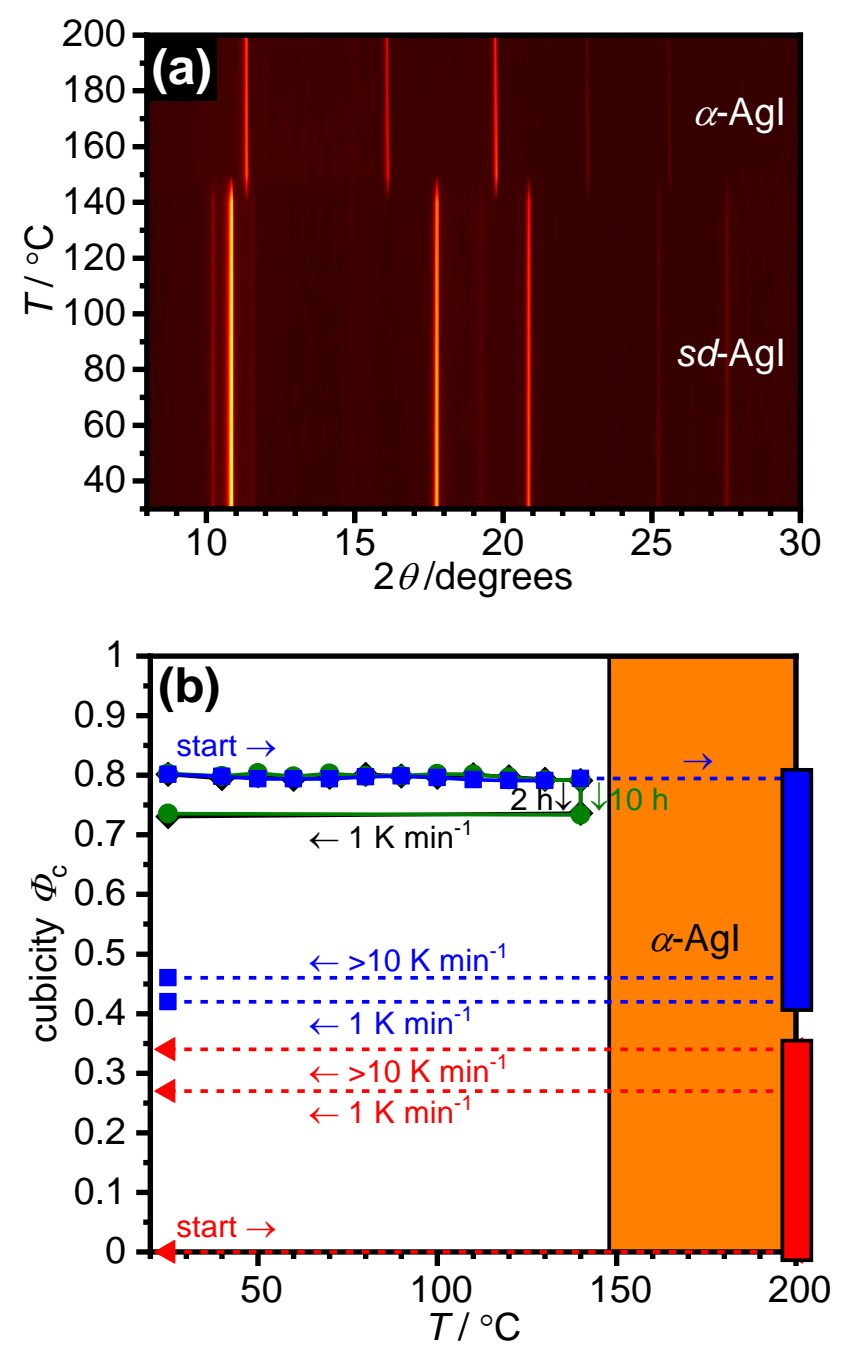

Figure 4 Memory effects in $\mathrm{AgI}$ after conversion to $\alpha$-AgI. (a) X-ray diffraction patterns (Mo $\mathrm{K} \alpha_{1}$ ) of the $s d$ - $\mathrm{AgI}$ to $\alpha$ - $\mathrm{AgI}$ phase transition collected upon heating to $200^{\circ} \mathrm{C}$. (b) Changes in cubicity upon heating $\beta$ - $\mathrm{AgI}$ and $s d$ - $\mathrm{AgI}$ with $\Phi_{\mathrm{c}}=0.80$ to $200^{\circ} \mathrm{C}$ followed by cooling at either 1 or $>10^{\circ} \mathrm{C} \mathrm{min}^{-1}$. Stacking disordered AgI with $\Phi_{\mathrm{c}}=0.80$ was also annealed at $140^{\circ} \mathrm{C}$ for either 2 or 10 hours, followed by cooling to room temperature at $1{ }^{\circ} \mathrm{C} \mathrm{min}^{-1}$.

The thermodynamic driving force towards $\beta$ - $\mathrm{AgI}$ is also illustrated by the fact that annealing $s d-$ AgI with $\Phi_{\mathrm{c}}=81 \%$ at $140^{\circ} \mathrm{C}$ for 2 hours leads to a decrease in cubicity of a few percent as shown 
in Figure 4(b). However, extending the annealing time to 10 hours at $140^{\circ} \mathrm{C}$ leads to the same cubicity illustrating that the decrease in cubicity reaches completion within the first two hours of annealing.

In summary, heating either fully hexagonal or highly cubic AgI to $\alpha$-AgI followed by cooling back leads to $s d-\mathrm{AgI}$ in both cases. As previously observed, ${ }^{45,50,51}$ there is indeed a memory effect in the sense that initially more cubic samples end up more cubic compared to samples that were originally more hexagonal. In addition to previously suggested origins of the memory effect mentioned earlier, ${ }^{45,50,51}$ a new explanation is put forward. From the crystallographic point of view, it is important to stress that $\beta$ - $\mathrm{AgI}$ is expected to be pyroelectric with crystals exhibiting a permanent net dipole moment. The point group related to its $P 6_{3} m c$ space group is $6 \mathrm{~mm}$, which is one of the ten polar crystallographic point groups. Fully cubic $\gamma$-AgI on the other hand is centrosymmetric and therefore by definition non-polar. For $s d-\mathrm{AgI}$, we have found $P 3 m 1$ as the space group, which is also polar with $3 m$ point group symmetry. While fully hexagonal $\beta$ - $\mathrm{AgI}$ is the most polar structure, the degree of polarity of $s d-\mathrm{AgI}$ is expected to decrease as its cubicity increases towards the non-polar $\gamma$-AgI. As it has been pointed out recently for polar ice structures, the overall energy of a pyroelectric materials is expected to be influenced by the dielectric properties of the medium surrounding the crystals. ${ }^{60}$ In case of the most polar $\beta$-AgI, it seems reasonable to suggest that some ions of opposite charge adsorb onto the polar crystal surfaces in order to stabilize them. Upon transformation to $a$-AgI, these excess ions can be expected to persist at their locations at least to some extent. Consequently, upon cooling the $\alpha$-AgI, they may provide a guiding influence towards the formation of more hexagonal structures in order to regain some of the original polarity and hence hexagonality of the sample.

\section{Conclusions}

The low-temperature phase of $\mathrm{AgI}$ at ambient pressure displays a strong tendency for stacking disorder. We suggest that $\beta$ - $\mathrm{AgI}, \gamma-\mathrm{AgI}$ and $s d$ - $\mathrm{AgI}$ should be used to describe the structures of the fully hexagonal, fully cubic and stacking disordered polytypes. Pure hexagonal $\beta$-AgI can only be prepared under iodide-rich precipitation conditions and the maximal cubicity obtained in our study was $81 \%$. In addition to investigating the effect of the $\mathrm{Ag}^{+}$to $\mathrm{I}^{-}$molar ratio on the cubicity of the $\mathrm{AgI}$ precipitates, the memory effect after transformation to $\alpha$ - $\mathrm{AgI}$ was confirmed and followed 
quantitatively. In addition to previous explanations, we suggested that the memory effect may be connected with the pyroelectric nature of $\beta$-AgI and the way the surfaces of the polar crystals are stabilized. In any case, the observed dependence of the cubicity of $\mathrm{AgI}$ on the $\mathrm{Ag}^{+}$to $\mathrm{I}^{-}$molar ratio upon precipitation now allows the targeted preparation of AgI samples with desired cubicities in the 0 to $81 \%$ range. To the best of our knowledge, this provides a first example of a system where the polytypism can be 'tuned' in a continuous fashion. If this design principle can be applied to other materials as well will be the topic of future investigations.

\section{Acknowledgements}

We thank the Royal Society for a University Research Fellowship (CGS, UF150665) and the UCL Chemistry Department for a DTP studentship (RLS). Furthermore, we are grateful to V. Molinero, T. Whale and S. Cox for helpful discussions.

\section{References}

(1) Li, H.; Yang, C.; Liu, F., Novel method for determining stacking disorder degree in hexagonal graphite by X-ray diffraction. Sci. China Chem. 2009, 52, 174-180.

(2) Warner, J. H.; Rümmeli, M. H.; Gemming, T.; Büchner, B.; Briggs, G. A. D., Direct Imaging of Rotational Stacking Faults in Few Layer Graphene. Nano Lett. 2009, 9, 102106.

(3) Moser, J.; Lévy, F., Random stacking in $\mathrm{MoS}_{2-\mathrm{x}}$ sputtered thin films. Thin Solid Films 1994, 240, 56-59.

(4) Kuhs, W. F.; Bliss, D. V.; Finney, J. L., High-Resolution Neutron Powder Diffraction Study of Ice I. J. Phys. Colloq., C1 1987, 48, 631-636.

(5) Malkin, T. L.; Murray, B. J.; Salzmann, C. G.; Molinero, V.; Pickering, S. J.; Whale, T. F., Stacking Disorder in Ice I. Phys. Chem. Chem. Phys. 2015, 17, 60-76.

(6) Playford, H. Y.; Whale, T. F.; Murray, B. J.; Tucker, M. G.; Salzmann, C. G., Analysis of stacking disorder in ice I using pair distribution functions. J. Appl. Crystal. 2018, 51, 12111220.

(7) Németh, P.; Garvie, L. A. J.; Aoki, T.; Dubrovinskaia, N.; Dubrovinsky, L.; Buseck, P. R., Lonsdaleite is faulted and twinned cubic diamond and does not exist as a discrete material. Nat. Comm. 2014, 5, 5447. 
(8) Salzmann, C. G.; Murray, B. J.; Shephard, J. J., Extent of Stacking Disorder in Diamond. Diam. Relat. Mat. 2015, 59, 69-72.

(9) Zimmermann, I.; Johnsson, M., Stacking faults in a layered cobalt tellurium phosphate oxochloride. Solid State Sci. 2015, 40, 67-70.

(10) Ainsworth, C. M.; Lewis, J. W.; Wang, C.-H.; Coelho, A. A.; Johnston, H. E.; Brand, H. E. A.; Evans, J. S. O., 3D Transition Metal Ordering and Rietveld Stacking Fault Quantification in the New Oxychalcogenides $\mathrm{La}_{2} \mathrm{O}_{2} \mathrm{Cu}_{2-4 x} \mathrm{Cd}_{2 x} \mathrm{Se}_{2}$. Chem. Mater. 2016, 28, 3184-3195.

(11) Willhammar, T.; Zou, X., Stacking disorders in zeolites and open-frameworks - structure elucidation and analysis by electron crystallography and X-ray diffraction. Z. Kristallogr. 2013, 228, 11 .

(12) Bond, A. D.; Boese, R.; Desiraju, G. R., On the Polymorphism of Aspirin: Crystalline Aspirin as Intergrowths of Two "Polymorphic" Domains. Angew. Chem. Int. Ed. 2007, 46, 618-622.

(13) Braun, D. E.; Gelbrich, T.; Kahlenberg, V.; Laus, G.; Wieser, J.; Griesser, U. J., Packing polymorphism of a conformationally flexible molecule (aprepitant). New J. Chem. 2008, $32,1677-1685$.

(14) Borodi, G.; Pop, M. M.; Onija, O.; Filip, X., Distinct Disordered Forms of Promethazine Hydrochloride: A Case of Intergrowth of Polymorphic Domains? Cryst. Growth Des. 2012, $12,5846-5851$.

(15) Price, S. L.; Braun, D. E.; Reutzel-Edens, S. M., Can computed crystal energy landscapes help understand pharmaceutical solids? Chem. Comm. 2016, 52, 7065-7077.

(16) Braun, D. E.; Lingireddy, S. R.; Beidelschies, M. D.; Guo, R.; Müller, P.; Price, S. L.; Reutzel-Edens, S. M., Unraveling Complexity in the Solid Form Screening of a Pharmaceutical Salt: Why so Many Forms? Why so Few? Cryst. Growth Des. 2017, 17, 5349-5365.

(17) Shilling, J. E.; Tolbert, M. A.; Toon, O. B.; Jensen, E. J.; Murray, B. J., Measurements of the Vapor Pressure of Cubic Ice and Their Implications for Atmospheric Ice Clouds. Geophys. Res. Lett. 2006, 33, L17801.

(18) Murray, B. J.; Salzmann, C. G.; Heymsfield, A. J.; Dobbie, S.; Neely, R. R.; Cox, C. J., Trigonal Ice Crystals in Earth's Atmosphere. Bull. Am. Met. Soc. 2015, 96, 1519-1531. 
(19) Carr, T. H. G.; Shephard, J. J.; Salzmann, C. G., Spectroscopic Signature of Stacking Disorder in Ice I. J. Phys. Chem. Lett. 2014, 5, 2469-2473.

(20) Behr, P.; Terziyski, A.; Zellner, R., Acetone Adsorption on Ice Surfaces in the Temperature Range T = 190-220 K: Evidence for Aging Effects Due to Crystallographic Changes of the Adsorption Sites. J. Phys. Chem. A 2006, 110, 8093-8107.

(21) Pan, Z.; Sun, H.; Zhang, Y.; Chen, C., Harder than diamond: superior indentation strength of wurtzite BN and lonsdaleite. Phys. Rev. Lett. 2009, 102, 055503.

(22) Quingkun, L.; Yi, S.; Zhiyuan, L.; Yu, Z., Lonsdaleite - a material stronger and stiffer than diamond. Scripta Mater. 2011, 65, 229-232.

(23) Tubandt, C.; Lorenz, F., Das elektrische Leitvermögen als Methode zur Bestimmung des Zustandsdiagramms binärer Salzgemische. Z. Phys. Chem. 1914, 87U, 543.

(24) Helmholz, L., The Crystal Structure of Hexagonal Silver Iodide. J. Chem. Phys. 1935, 3, 740-746.

(25) Burley, G., Structure of Hexagonal Silver Iodide. J. Chem. Phys. 1963, 38, 2807-2812.

(26) Wilsey, R. B., XXXI. The crystal structure of the silver halides. Phil. Mag. 1921, 42, 262263.

(27) Prager, P., Polytypism in silver iodide. Acta Cryst. 1974, A30, 369-373.

(28) Prager, P., Study of a mixed-polytype crystal of silver iodide containing a new $12 \mathrm{H}$ polytype. Acta Cryst. 1977, A33, 25-28.

(29) Prager, P. R., Growth and characterization of AgI polytypes. Prog. Crystl. Growth Charact. Mater. 1983, 7, 451-492.

(30) Germann, F. E. E.; Hylan, M. C., THE PHOTOGRAPHIC SENSITIVENESS OF SILVER IODIDE. J. Am. Chem. Soc. 1923, 45, 2486-2493.

(31) Vonnegut, B., The Nucleation of Ice Formation by Silver Iodide. J. Appl. Phys. 1947, 18, 593-595.

(32) Vonnegut, B.; Chessin, H., Ice Nucleation by Coprecipitated Silver Iodide and Silver Bromide. Science 1971, 174, 945-946.

(33) Marcolli, C.; Nagare, B.; Welti, A.; Lohmann, U., Ice nucleation efficiency of AgI: review and new insights. Atmos. Chem. Phys. 2016, 16, 8915-8937. 
(34) Feng, S.; Xu, H.; Liu, L.; Song, Y.; Li, H.; Xu, Y.; Xia, J.; Yin, S.; Yan, J., Controllable synthesis of hexagon-shaped $\beta$-AgI nanoplates in reactable ionic liquid and their photocatalytic activity. Colloids Surf. A 2012, 410, 23-30.

(35) Jiang, W.; An, C.; Liu, J.; Wang, S.; Zhao, L.; Guo, W.; Liu, J., Facile aqueous synthesis of $\beta$-AgI nanoplates as efficient visible-light-responsive photocatalyst. Dalton Trans. 2014, 43, 300-305.

(36) An, C.; Liu, J.; Wang, S.; Zhang, J.; Wang, Z.; Long, R.; Sun, Y., Concaving AgI submicroparticles for enhanced photocatalysis. Nano Energy 2014, 9, 204-211.

(37) An, C.; Wang, J.; Liu, J.; Wang, S.; Zhang, Q.-H., Plasmonic enhancement of photocatalysis over Ag incorporated AgI hollow nanostructures. RSC Adv. 2014, 4, 2409 2413.

(38) An, C.; Wang, S.; Sun, Y.; Zhang, Q.; Zhang, J.; Wang, C.; Fang, J., Plasmonic silver incorporated silver halides for efficient photocatalysis. J. Mat. Chem. A 2016, 4, 43364352.

(39) Kalnina, D.; Gross, K. A.; Onufrijevs, P.; Dauksta, E.; Nikolajeva, V.; Stankeviciute, Z.; Kareiva, A., The Antimicrobial Action of Silver Halides in Calcium Phosphate. Key Eng. Mater. 2015, 631, 384-389.

(40) Tatsumisago, M.; Shinkuma, Y.; Minami, T., Stabilization of superionic $\alpha$-Agl at room temperature in a glass matrix. Nature 1991, 354, 217-218.

(41) Makiura, R.; Yonemura, T.; Yamada, T.; Yamauchi, M.; Ikeda, R.; Kitagawa, H.; Kato, K.; Takata, M., Size-controlled stabilization of the superionic phase to room temperature in polymer-coated AgI nanoparticles. Nat. Mat. 2009, 8, 476-480.

(42) Funke, K.; Banhatti, R. D.; Grabowski, P.; Nowinski, J.; Wrobel, W.; Dinnebier, R.; Magdysyuk, O., Low-temperature $\alpha$-AgI confined in glass: Structure and dynamics. Solid State Ionics 2015, 271, 2-9.

(43) Zhang, H.; Tsuchiya, T.; Liang, C.; Terabe, K., Size-Controlled AgI/Ag Heteronanowires in Highly Ordered Alumina Membranes: Superionic Phase Stabilization and Conductivity. Nano Lett. 2015, 15, 5161-5167.

(44) Yamamoto, T.; Maesato, M.; Hirao, N.; Kawaguchi, S. I.; Kawaguchi, S.; Ohishi, Y.; Kubota, Y.; Kobayashi, H.; Kitagawa, H., The Room-Temperature Superionic 
Conductivity of Silver Iodide Nanoparticles under Pressure. J. Am. Chem. Soc. 2017, 139, 1392-1395.

(45) Bloch, R.; Möller, H., Über die Modifikationen des Jodsilbers. Z. Phys. Chem. 1931, 152A, 245.

(46) Mills, D. R.; Perrott, C. M.; Fletcher, N. H., The production of single crystals of AgI. J. Cryst. Growth 1970, 6, 266-268.

(47) Cochrane, G., Preparation of single crystals of hexagonal silver iodide. Brit. J. Appl. Phys. 1967, $18,687$.

(48) Verwey, E. J. W.; Kruyt, H. R., Zur Kenntnis der elektrischen Doppelschicht bei Kolloiden. II. Z. Phys. Chem. 1933, 167A, 137.

(49) Kolkmeijer N, H.; Hengel, J. W. A. v., Über das reguläre und das hexagonale Silberjodid. Z. Krist. 1934, 88, 317.

(50) Burley, G., The 'memory effect' in silver iodide. Acta Cryst. 1967, 23, 1-5.

(51) Maskasky, J. E., High phase-transition temperature for $\beta$-AgI to $\alpha$-AgI and an explanation of the memory effect. Phys. Rev. B 1991, 43, 5769-5772.

(52) Strock, L. W., Kristallstruktur des Hochtemperatur-Jodsilbers $\alpha$-AgI. Z. Phys. Chem. 1934, $25 B, 441$.

(53) Stephen, H., Superionics: crystal structures and conduction processes. Rep. Prog. Phys. 2004, 67, 1233.

(54) Treacy, M. M. J.; Newsam, J. M.; Deem, M. W., A General Recursion Method for Calculating Diffracted Intensities From Crystals Containing Planar Faults. Proc. Roy. Soc. Lond. 1991, A433, 499-520.

(55) Larsen, A. C.; Von Dreele, R. B. GSAS - General Structure Analysis System, University of California: 1985.

(56) Salzmann, C. G.; Murray, B. J.; Shephard, J. J., Extent of stacking disorder in diamond. Diamond Relat. Mater. 2015, 59, 69-72.

(57) Malkin, T. L.; Murray, B. J.; Brukhno, A. V.; J., A.; Salzmann, C. G., Structure of Ice Crystallized from Supercooled Water. Proc. Natl. Acad. Sci. USA 2012, 109, 1041-1045.

(58) Mellander, B. E.; Bowling, J. E.; Baranowski, B., Phase Diagram of Silver Iodide in the Pressure Range 2.5-10 kbar and the Temperature Range 4-330 ${ }^{\circ}$ C. Phys. Scr. 1980, 22, 541. 
(59) Hull, S.; Keen, D. A., Pressure-induced phase transitions in AgCl, AgBr, and AgI. Phys. Rev. B 1999, 59, 750-761.

(60) Del Ben, M.; VandeVondele, J.; Slater, B., Periodic MP2, RPA, and Boundary Condition Assessment of Hydrogen Ordering in Ice XV. J. Phys. Chem. Lett. 2014, 5, 4122-4128. 


\section{For Table of Contents Use Only}

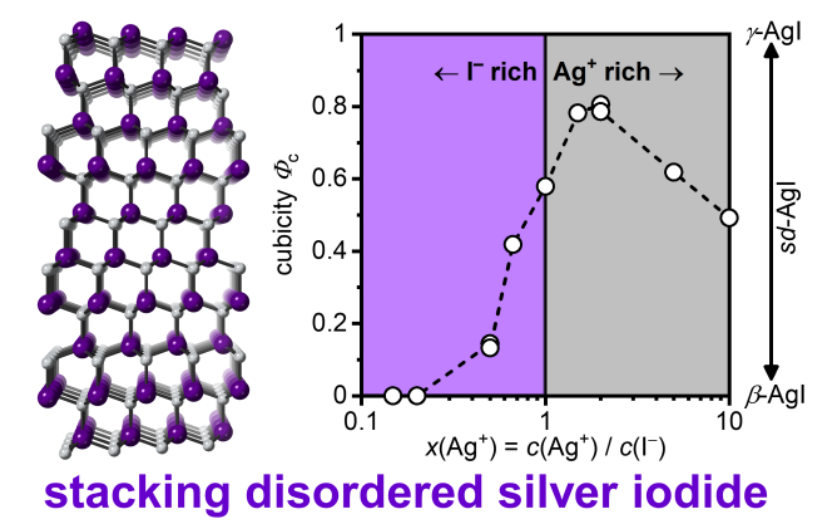

Silver iodide samples ranging from fully hexagonal to $81 \%$ cubic can be obtained by tuning the cation to anion ratio upon precipitation. Furthermore, we follow the well-known memory effect of silver iodide, observed after conversion to the superionic high-temperature phase, quantitatively and provide a new explanation based on surface effects arising from the stabilization of the pyroelectric hexagonal silver iodide. 